\title{
BIOSSÍNTESE, CARACTERIZAÇÃO E ATIVIDADE FOTOCATALÍTICA DE NANOPARTÍCULAS DE TITÂNIO A PARTIR DA Aloe vera
}

\author{
Pâmela Muraro1; Virginia Rech²; William Leonardo da Silva ${ }^{3}$
}

\section{RESUMO}

A nanotecnologia verde baseia-se na aplicação de sistemas nanoestruturados, principalmente nanopartículas metálicas, relacionando com o desenvolvimento sustentável. Opresente trabalho tem como objetivo síntetizar, caracterizar e avaliar a atividade fotocatalítica de nanopartículas de titânio (TiNPs), a partir do extrato de Aloe vera, para a remoção do corante RhB. Para a síntese das TiNPs, foi utilizado o método de biorredução, sendo caracterizadas por porosimetria de nitrogênio e potencial zeta. Assim, as TiNPs apresentaram área superficial de $118,38 \mathrm{~m}^{2} \mathrm{~g}^{-1}$, diâmetro de poros de $9,2 \mathrm{~nm}$ e volume de poros de $0,2 \mathrm{~cm}^{3} \mathrm{~g}^{-1}$, e uma carga superficial de $-4,90 \mathrm{mV}$. Os ensaios de fotocatálise, TiNPs apresentaram uma remoção de $33,66 \%$ do corantes RhB, com uma velocidade cinética de $0,0039 \mathrm{~min}^{-1}$, sob radiação visível após 90 minutos $\left(\mathrm{pH}=4,03\right.$, [TiNPs] $\left.=0,7 \mathrm{gL}^{-1} \mathrm{e}[\mathrm{RhB}]=20 \mathrm{mgL}^{-1}\right)$. Por conseguinte, as TiNPs apresentaram propriedades promissoras como materiais alternativos para aplicação no tratamento de águas residuárias com corantes.

Palavras-chave: Nanobiotecnologia; Rodamina B; Síntese verde; Tratamento de Águas Residuárias.

Eixo Temático: Tecnologia, inovação e desenvolvimento sustentável - TIDS

\section{INTRODUÇÃO}

Nas últimas décadas, o desenvolvimento populacional trouxe consigo a crescente demanda de bens de consumo, fato este que causou a explosão industrial de diversos seguimentos comerciais, principalmente o setor têxtil responsável pela geração considerável de grandes volumes de efluentes com corantes (ALMEIDA; DILARRI; CORSO, 2019). Estas águas residuárias coloridas, quando incorretamente dispostas podem causar sérios problemas ambientais (GARCIA, 2006), provocando alterações como a poluição visual e a modificação dos ciclos biológicos, afetando os

\footnotetext{
1Doutorando do Programa de Pós-Graduação em Nanociências, Universidade Franciscana, pemuraro@gmail.com;

2 Coorientadora - Professora do Programa de Pós-Graduação em Nanociências, Universidade Franciscana,vga.cielo@gmail.com;

${ }^{3}$ Orientador - Professor do Programa de Pós-Graduação em Nanociências, Universidade Franciscana, w.silva@ufn.edu.br.
} 
seres aquáticos e prejudicando os processos de fotossíntese (ZANONI; CARNEIRO, 2001).

Assim, cerca de 10.000 (dez mil) corantes e pigmentos estão disponíveis para a indústria têxtil, estimando que a produção mundial esteja em torno de $8.10^{5}$ ton./ano, e cerca de 10 - 15\% dos corantes se tornam biodisponíveis por meio das águas residuárias (NIEBISCH et al., 2014). Por isso, encontra-se uma intensa dificuldade no tratamento final de efluentes que contém estes corantes de natureza sintética e a presença de muitos anéis aromáticos na estrutura molecular fazem dos corantes compostos com uma elevada estabilidade química e de difícil biodegradação (NGUYEN; JUANG, 2013).

Os Processos Oxidativos Avançados (POAs) são tecnologias eficientes, que envolvem a geração de radical hidroxila $\left({ }^{\circ} \mathrm{OH}\right)$, altamente oxidante e seletivo, sendo capaz de degradar uma série de moléculas recalcitrantes em compostos biodegradáveis ou promover a mineralização completa em $\mathrm{CO}_{2}, \mathrm{H}_{2} \mathrm{O}$, por meio de uma série de reações de oxirredução (MIKLOS et al., 2018).

Dentro dos POAs, a fotocatálise heterogênea destaca-se pois se baseia na separação de cargas elétricas em sólidos específicos (semicondutores), por meio da excitação luminosa, possibilitando reações de oxidação-redução na superfície deste semicondutor (COMPARELLI et al., 2005). Estes semicondutores caracterizam-se por apresentar duas regiões de energia, sendo: uma de mais baixa energia, denominada a banda de valência (BV) e outra de maior energia, chamada banda de condução (BC). Além disso, a região entre estas bandas é denominada banda proibida ou band gap (GOGATE; PANDIT, 2004), que forma uma vacância positiva na banda de valência $\left(\mathrm{h}^{+} \mathrm{BV}\right)$, que serão responsáveis pela formação de radicais hidroxilas $\left({ }^{\circ} \mathrm{OH}\right)$ (CHATTERJEE; DASGUPTA, 2005). Desse modo, a eficiência do processo fotocatalítico depende diretamente da competição entre o elétron que foi retirado da superfície do semicondutor e as possíveis recombinações do par elétron/vacância.

Com o advento da nanotecnologia verde, que é uma abordagem entre sistemas nanoestruturados e o desenvolvimento sustentável, vem sendo estudado métodos alternativos ambientalmente corretos e com baixo custo, como os processos de biossíntese a partir de biomoléculas e microrganismos (NARAYANAN; SAKTHIEVEL, 
2015), destacando-se as nanopartículas metálicas verdes com propriedades específicas, para aplicação na fotocatálise verde (DA SILVA et al., 2017).

A maioria das nanopartículas metálicas obtidas por meio de rotas de síntese verde apresenta como características almejáveis, sob o ponto de vista de sustentabilidade o fato de serem ecoamigáveis (utilizam solventes menos tóxicos), biocompatíveis (podem ser empregadas diretamente a organismos-alvo), simples (número de etapas menor), biodegradáveis (podem ser degradadas por rotas biológicas) e rendimento alto (SHARMA; YNGARD; LIN; 2009; THAKKANA; MHATRE; PARIK, 2010).

Neste contexto, o presente trabalho tem como objetivo sintetizar nanopartículas de titânio (TiNPs), a partir do extrato da Aloe vera, para aplicação como catalisador na remoção do corante Rodamina $B(R h B)$, sob radiação visível.

\section{METODOLOGIA}

\subsection{PREPARAÇÃO DO EXTRATO DA Aloe vera}

Inicialmente, para a preparação do extrato da Aloe vera, foi realizada a separação da casca da folha para remoção do gel (seiva), onde foi fervido por 30 minutos em 1000 mL de água destilada estéril.

\subsection{BIOSSÍNTE DE NANOPARTÍCULAS DE TITÂNIO}

A síntese das nanopartículas de titânio foi realizada pelo método biorredução, conforme adaptação da literatura (RAO et al., 2015). Assim, como precursor de titânio foi utilizado o isopropóxido de titânio $\left(0,25\right.$ mol.L-1, $\mathrm{C}_{12} \mathrm{H}_{28} \mathrm{O}_{4} \mathrm{Ti}$, Sigma-Aldrich, 97\%) em contato com $150 \mathrm{~mL}$ do extrato de Aloe vera, sob agitação magnética (240 minutos e $250 \mathrm{rpm})$, sob temperatura ambiente $\left(25 \pm 2^{\circ} \mathrm{C}\right)$. Em seguida, a solução foi seca na estufa (DeLeon, modelo único) por 8 horas / $100^{\circ} \mathrm{C}$, sendo nomeada TiNPs.

\subsection{TÉCNICAS DE CARACTERIZAÇÃO}


Para as análises da amostra sintetizada foi utilizado a porosimetria de $\mathrm{N}_{2}$, aonde as áreas específicas (SBET) foram determinadas pela Equação de BrunauerEmmett-Teller (Método BET), na faixa de $\mathrm{P} \mathrm{Po}^{-1}=0,05$ a 1,0, enquanto o diâmetro e volume de poros pela Equação de Barret-Joyner-Halenda (Método BJH). Previamente, as amostras (100-200 mg) foram desgaseificadas sob uma pressão de $10^{-2}$ mbar com temperatura de $120 \stackrel{\circ}{ } \mathrm{C}$ durante $12 \mathrm{~h}$, em um equipamento Asap 2020 da Micromeritics ${ }^{\circledR}$. (THOMMES et al., 2015). Para o potencial zeta (PZ), foi utilizado o equipamento da marca Malvern-Zetasizer®) modelo nanoZS (ZEN3600, Reino Unido) com células capilares fechadas (DTS 1060) (Malvern, Reino Unido) para medir a carga superficial da amostra.

\subsection{ATIVIDADE FOTOCATALÍTICA}

Os ensaios de degradação foram realizados em um reator batelada, com catalisador em suspensão (slurry). O volume reacional utilizado foi de $100 \mathrm{~mL}$, conforme a literatura (MURARO et al., 2020), com pH =4,3, concentração do catalisador de $0,7 \mathrm{~g} \cdot \mathrm{L}^{-1}$, concentração do poluente orgânico de $20 \mathrm{mg} \cdot \mathrm{L}^{-1}$ e tempo de reação de 90 minutos.

\subsubsection{Cinética de degradação}

O modelo de Langmuir-Hinshelwood ( $\mathrm{L}-\mathrm{H})$ identifica três etapas principais de uma reação heterogênea, sendo (KONSTANTINOU; ALBANIS, 2004; GAYA; ABDULLAH, 2008) adsorção do reagente na superfície do catalisador, reação superficial e dessorção dos produtos, sendo a etapa de reação superficial mais lenta e responsável pela cinética de reação. A Equação (1), proposta por L-H, estabelece o mecanismo de reação em que coexistem dois parâmetros: pré-equilíbrio de adsorção e reação superficial lenta. Matematicamente, a taxa de reação observada apresenta uma dependência com as concentrações do soluto, conforme a Eq. (1):

$$
-r_{i}=-\frac{d C_{i}}{d t}=\frac{k_{s} \cdot K \cdot C_{i}}{1+K \cdot C_{i}}
$$




\section{Onde:}

-ri = taxa da reação $\left(\mathrm{mol}_{\text {tempo }}^{-1}\right.$ volume $\left.^{-1}\right)$;

$k_{s}=$ velocidade específica da reação superficial;

$\mathrm{Ci}=$ concentração do reagente $\left(\mathrm{mol}_{\text {volume }}{ }^{-1}\right)$;

$K=$ constante de adsorção do reagente na superfície do catalisador.

Para sistemas onde o reagente encontra-se suficientemente diluído o termo KCi no denominador da Equação (1) é muito menor que 1, podendo ser desprezado. Desta maneira obtêm-se a Equação (2) que, quando integrada gera a equação (3).

$$
\begin{gathered}
-r_{i}=-\frac{d C_{i}}{d t}=k_{s} . K . C_{i} \\
\ln \left(\frac{C_{i o}}{C_{i}}\right)=k_{s} . K . t=k . t \text { ou } C_{i}=C_{i o} \cdot e^{-k t}
\end{gathered}
$$

$\mathrm{Na}$ Equação (3), as constantes de reação superficial $\left(k_{s}\right)$ e de equilíbrio de adsorção $(K)$ são contidas em uma única constante $\mathrm{k}$, denominada de constante cinética aparente da reação. O gráfico de $\ln \mathrm{C}_{\mathrm{i} 0} \mathrm{C}_{\mathrm{i}}^{-1}$ versus o tempo representa uma linha reta, sendo $\mathrm{k}$ dado pelo coeficiente angular.

\section{RESULTADOS E DISCUSSÃO}

\subsection{CARACTERIZAÇÃO DAS NANOPARTÍCULAS DE TITÂNIO}

A Tabela 1 apresenta os resultados da porosimetria de $\mathrm{N}_{2}$ com área específica (SBET), diâmetro de poros (Dp) e o volume de poro (Vp) da TiNPs. Para fins comparativos, utilizou-se o catalisador comercial dióxido de titânio ( $\left.\mathrm{TiO}_{2}-\mathrm{P} 25\right)$ (DA SILVA et al., 2016).

Tabela 1. Área de superfície ( $\left.\mathrm{S}_{\mathrm{BET}}\right)$, volume de poro $(\mathrm{Vp})$, diâmetro de poro (Dp) e potencial zeta (PZ) do $\mathrm{TiO}_{2}(\mathrm{P} 25)$ e TiNPs

\begin{tabular}{ccccc}
\hline Amostra & $\mathbf{S}_{\mathrm{BET}}\left(\mathbf{m}^{\mathbf{2}} \mathbf{g}^{-1}\right)$ & $\mathbf{V p}\left(\mathbf{c m}^{\mathbf{3}} \mathbf{g}^{-1}\right)$ & $\mathbf{D p} \mathbf{( n m})$ & $\mathbf{P Z}(\mathbf{m V})$ \\
\hline $\mathrm{TiO}_{2}(\mathrm{P} 25)$ & $56 \pm 0.07$ & $0.07 \pm 0.04$ & $4.8 \pm 0.03$ & $-24.00 \pm 0.50$ \\
\hline $\mathrm{TiNPs}$ & $118.38 \pm 0.05$ & $0.2 \pm 0.03$ & $9.2 \pm 0.04$ & $-4.90 \pm 0.30$ \\
\hline
\end{tabular}


Fonte: Construção do Autor.

Conforme a Tabela 1, a amostra TiNPs apresentou maior valor de diâmetro do poro $\mathrm{Dp}(9,2 \mathrm{~nm})$ comparado ao $\mathrm{TiO}_{2}$ com 4,8 nm, como características de materiais mesoporosos (IUPAC, 1972). Além disso, as TiNPs apresentaram um volume de poros cerca de $185 \%$ maior que o catalisador comercial, bem como uma área superficial (SBET) cerca de $111 \%$ maior que do $\mathrm{TiO}_{2}$ (P25). Para fotocatálise heterogênea, o ideal é que o nanocatalisador apresente uma área específica elevada para promover mais adsorção do poluente a ser degradado, bem como uma porosidade significativamente grande para promover a difusão do poluente para a superfície catalítica do nanocatalisador (LIU et al., 2008). Na análise de PZ., as nanopartículas de titânio sintetizadas pelo processo de biossíntese apresentaram uma carga superficial negativa, favorecendo a interações eletrostática com o corante RhB, em virtude da compatibilidade de carga com o corante (origem catiônica) (GOSWAMI; PHUKAN, 2017).

A Figura 1, as isotermas de adsorção/dessorção de $\mathrm{N}_{2}$ indicaram uma isoterma do tipo $V$, típica de materiais mesoporosos, com preenchimento multicamadas (MCCUSKER et al., 2001). Além disso, as TiNPs apresentaram uma curva de histerese tipo $\mathrm{H} 1$, comumente observada em materiais porosos caracterizados por serem constituídos por aglomerados ou esferas uniformes compactadas, na forma de cilindros com extremidades abertas (THOMMES et al., 2015).

Figura 1. Isotermas de adsorção/dessorção de $\mathrm{N}_{2}$ das TiNPs sintetizadas pelo processo de biossíntese 


\section{QUFN}

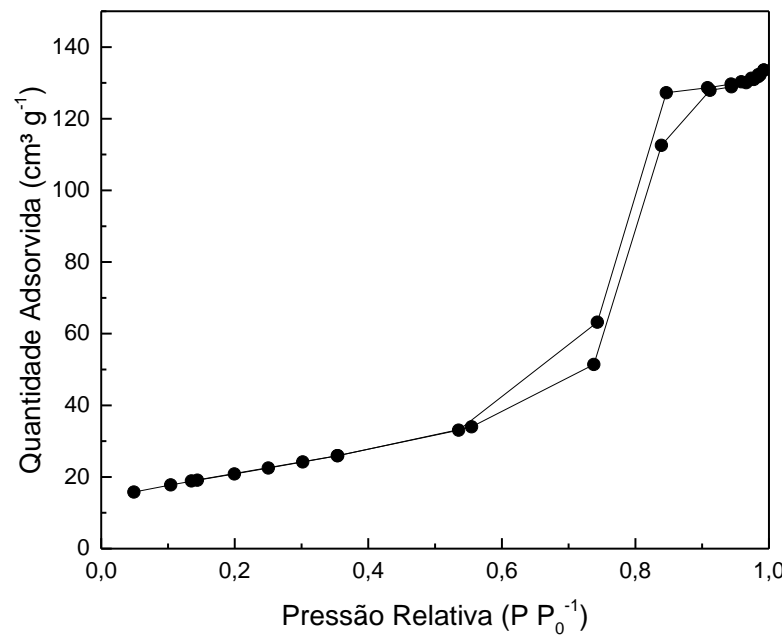

Fonte: Construção do autor.

\subsection{ATIVIDADE FOTOCATALÍTICA}

A Figura 2 apresenta a velocidade específica de degradação do corante $R h B$ utilizando TiNPs, sob a radiação visível, após 90 minutos de reação.

Figura 2. Velocidade específica de reação de degradação do corante RhB sob radiação visível após 90 minutos de reação ([TiNPs] $=0,7 \mathrm{~g} \mathrm{~L}^{-1},[\mathrm{RhB}]=20 \mathrm{mg} \mathrm{L}^{-1}, \mathrm{pH}=4,03$ e T $=25^{\circ} \mathrm{C}$ )

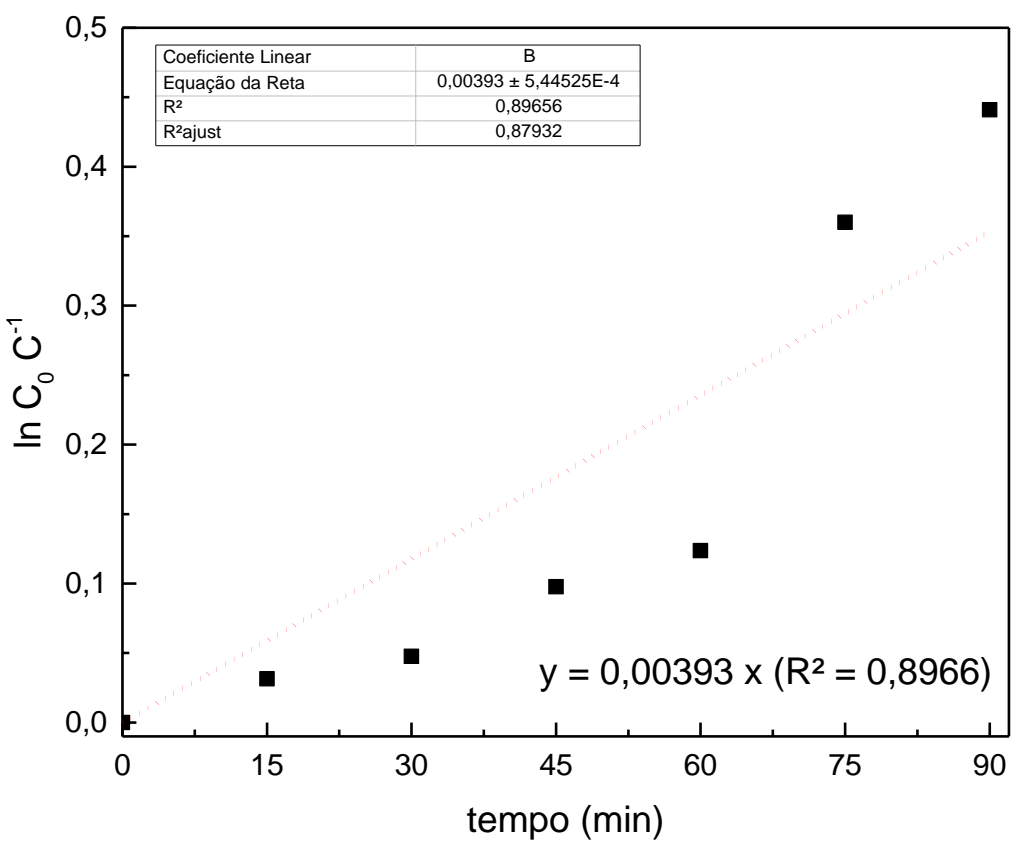


Fonte: Construção do autor.

Conforme a Figura 2, as TiNPs apresentaram um comportamento cinético de pseudo primeira-ordem com uma remoção de 33,66\%, após 90 minutos, e uma velocidade específica $\mathrm{k}=0,0039 \mathrm{~min}^{-1}$. Destaca-se que o catalisador comercial $\mathrm{TiO}_{2}$, apresentou degradação menor do que o $\mathrm{TiO}_{2}$ (P25) $\left(42,14 \%, \mathrm{k}=0,0043 \mathrm{~min}^{-1}\right)$ (DA SILVA et al., 2016), fato que pode ser justificado por meio da sua carga superficial (4,90 mV) ser menor do que o catalisador comercial (-24,0 mV), assim proporcionando uma menor interação entre a nanopartícula e a RhB.

\section{CONCLUSÃO}

De acordo com este estudo, foi possível verificar o sucesso da síntese de TiNPs a partir do extrato de Aloe vera, atuando como agente redutor pelo método de redução química. A análise de porosimetria de $\mathrm{N}_{2}$ comprovou o tamanho nanométrica da amostra sintetizada tendo como resultado uma alta área de superfície comparado ao catalisador comercial $\mathrm{TiO}_{2}$ (P25). O diâmetro de poros foi classificado como mesoporos com isoterma $\mathrm{V}$ e histerese $\mathrm{H} 1$. Na fotocatálise, a degradação da RhB foi de $33,66 \%$ na luz visível, com $k=0,0039 \mathrm{~min}^{-1}$. Assim conclui-se que as TiNPs são nanopartículas promissores para o uso fotocatalítico para a degradação de poluentes orgânicos como a RhB desde que seja realizado ensaios preliminares das condições ideias para o processo de fotocatálise

\section{AGRADECIMENTOS}

Os autores gostariam de agradecer a Universidade Franciscana (UFN), Programa de Pós-graduação em Nanociências e a CAPES (Coordenação de Aperfeiçoamento de Pessoal de Nível Superior) pelo suporte para a realização do presente trabalho.

\section{REFERÊNCIAS}


ALMEIDA, E. J. R.; DILARRI, G.; CORSO C. R. A indústria têxtil no Brasil: Uma revisão dos seus impactos ambientais e possíveis tratamentos para os seus efluentes. Departamento de Bioquímica e Microbiologia, Universidade Estadual Paulista, Rio Claro, SP. P. 1-18, 2015.

CHATTERJEE, D.; DASGUPTA, S. Visible light induced photocatalytic degradation of organic pollutants. Journal of Photochemistry and Photobiology C:

Photochemistry Reviews, v. 6, p. 186-205, 2005.

COMPARELLI, R.; FANIZZA, E.; CURRI M. L.; COZZOLI, P. D.; MASCOLO, G.; AGOSTIANO, A. UV-induced photocatalytic degradation of azo dyes by organiccapped $\mathrm{ZnO}$ nanocrytals immobilized onto substrates. Applied Catalysis B: Environmental, v. 60, p. 1-11, 2005.

DA SILVA, W. L.; LANSARIN, M.; DOS SANTOS, J. H. Z.; SILVEIRA, F. Photocatalytic degradation of rhodamine $B$, paracetamol and diclofenac sodium by supported titania-based catalysts from petrochemical residue: effect of doping with magnesium. Water Science and Technology, v. 74, p. 2370-2383, 2016. DA SILVA, L. P.; BONATTO, C.; PEREIRA, F.; SILVA, L.; ALBERNAZ, V. L. Nanotecnologia verde para síntese de nanopartículas metálicas. Biotecnologia Aplicada à Agro\&Indústria, v. 4, p. 967-1011, 2017.

GARCIA, J. C.. Degradação fotocatalítica artificial e solar de efluentes têxteis por processos oxidativos avançados utilizando $\mathrm{TiO}_{2}$. 2006.180 p. Tese (Doutorado em Química) - Programa de Pós-Graduação em Química do Departamento de Química do Centro de Ciências Exatas, a Universidade Estadual de Maringá, 2006.

GAYA, U.I.; ABDULLAH, A.H. Heterogeneous photocatalytic degradation of organic contaminants over titanium dioxide: A review of fundamentals, progress and problems. Journal of Photochemistry and Photobiology C: Photochemistry Reviews, v. 9, p. 1-12, 2008.

GOGATE, P.R.; PANDIT, A.B. A review of imperative technologies for wastewater treatment I. Oxidation technologies at ambiental conditions. Advanced

Environmental Research, v. 8, p. 501-551, 2004. 
IUPAC. Manual of symbols and Terminology. Pure Apllied Chemical, v. 31, p. 578, 1972.

GOSWAMI, M.; PHUKAN, P. Enhanced adsorption of cationic dyes using sulfonic acid modified activated carbon.Journal of Environmental Chemical Engineering, v. 5, n. 4, p. 3508-3517, 2017.

KONSTANTINOU, I. K.; ALBANIS, T. A. Photocatalytic transformation of pesticides in aqueous titanium dioxide suspensions using artificial and solar light: intermediates and degradation pathways. Applied Catalysis B: Environmental, v. 42, p. 319-335, 2003.

LIU, Y; WANG, X.; YANG, F.;YANG, X. Excellent antimicrobial properties of mesoporous anatase $\mathrm{TiO}_{2}$ and $\mathrm{Ag} / \mathrm{TiO}_{2}$ composite films. Microporous and Mesoporous Materials, v. 114, p. 431-439, 2008.

MIKLOS, D.; REMY, C.; JEKEL, M.; LINDEN, K. G.; DREWES, J. E.; HÜBNER, U. Evaluation of advanced oxidation processes for water and wastewater treatment-A critical review. Water research, v. 139, p. 118-131, 2018.

MCCUSKER, L. B.; LIEBAU, F.; ENGELHARDT, G. Nomenclature of structural and compositional characteristics of ordered microporous and mesoporous materials with inorganic hosts (IUPAC Recommendations 2001). Pure and Applied Chemistry, v. 73, n. 2, p. 381-394, 2001.

NARAYANAN, K B; SAKTHIVEL, N. Green synthesis of biogenic metal nanoparticles by terrestrial and aquatic phototrophic and heterotrophic eukaryotes and biocompatible agents. Advances in colloid and interface science, v. 169, n. 2, p. 59-79, 2011.

NGUYEN, T. A.; JUANG, R-S.. Treatment of waters and wastewaters containing sulfur dyes: a review. Chemical engineering journal, v. 219, p. 109-117, 2013. RAO, K.; ASHOK, C. H.; RAO, K. V.; CHAKRA, C. S.; RAJENDAR, V. Ganapathi. Synthesis of $\mathrm{TiO}_{2}$ nanoparticles from orange fruit waste. Synthesis, v. 2, n. 1, p. 1, 2015.

NIEBISCH, C. H.; Foltran, C.; Domingues, R. C. S.; Paba, J. Assessment of Heteroporus biennis secretion extracts for decolorization of textile dyes.

International Biodeterioration \& Biodegradation, v. 88, p. 20-28, 2014. 
SHARMA, V. K.; YNGARD, R. A.; LIN, Y. Silver nanoparticles: green synthesis and their antimicrobial activities. Advances in Colloid and Interface Science, v. 145, p. 83-96, 2009.

THAKKAR, K. N.; MHATRE, S. S.; PARIKH, R. Biological synthesis of metallic nanoparticles. Nanomedicine: nanotechnology, biology and medicine, v. 6, p. 257-262, 2010

THOMMES, M.; Kaneko, K.; Neimark, A. V.; Olivier, J. P.; Rodriguez-Reinoso, F.; Rouquerol, J. Sing, K. S. Physisorption of gases, with special reference to the evaluation of surface area and pore size distribution (IUPAC Technical Report). Pure and applied chemistry, v. 87, n. 9-10, p. 1051-1069, 2015.

ZANONI, M.V.B.; CARNEIRO, P.A. O descarte dos corantes têxteis. Ciência Hoje, v. 29, n. 174, p. 61-71, 2001. 